\title{
Application of Blockchain Technology in Online Education
}

\author{
https://doi.org/10.3991/ijet.v13i10.9455 \\ Han Sun ${ }^{(\varpi)}$ \\ The Army Infantry Academy of PLA, Shijiazhuang, China \\ dollarsunhan@126.com \\ Xiaoyue Wang \\ Shijiazhuang Preschool Teachers College, Shijiazhuang, China \\ Xinge Wang \\ National Defense University of PLA, Beijing, China
}

\begin{abstract}
Blockchain is a data structure of data blocks arranged in chronological order. It is featured by decentralization, trustworthiness, data sharing, security, etc. It has been widely used in digital currency, smart contract, credit encryption and other fields. With the development of the Internet technology, online education, a novel education mode, has been greatly popularized. However, this education mode still faces many problems in course credibility, credit and certificate certification, student privacy, and course sharing. Through literature review and case analysis, this paper discusses the basic technical principles and application features of blockchain technology, and proposes a solution to the problems of online education based on blockchain technology. The blockchain technology can store learning records in a trusted, distributed manner, provide credible digital certificates, realize learning resource sharing with smart contract, and protect intellectual property through data encryption. The research shows that the integration of blockchain technology is a promising trend in the development of online education.
\end{abstract}

Keywords-Blockchain; online education; digital certificate; smart contract

\section{Introduction}

Owing to the rapid development of Internet technology, online education has entered a stage of rapid growth since the turn of the century. Online education, also known as distance education or online learning, is a web-based teaching method for content dissemination and fast learning using information technology and Internet technology. With the Internet as the medium, online teaching transcends the limits on venue, environment, time and teachers, and offers students quality teaching activities anytime, anywhere.

The various types of online education can be divided into vocational training, examination and certification training, personal skills improvement, language education, 
early childhood education, K-12 education (kindergarten to 12 th grade), etc. In China, online education platforms are springing up thanks to the efforts by players like traditional Internet giants (e.g. NetEase and Tencent), traditional education companies (e.g. New Oriental, Xueersi and Hujiang), universities, as well as primary and secondary schools. In recent years, massive open online courses (MOOCs) has been brought to public attention. Originated in the US, MOOCs are developed by leading content providers like Coursera, Udacity and edX. Since 2012, top American universities have been setting up online learning platforms and offering free online courses. Targeting higher education, MOOCs are featured by quality teaching programs and independent management systems.

Despite the immense popularity, the current forms and systems of online education have many defects in the face of an increasingly open and digital Internet. For example, the learning process and results of MOOCs are short of public recognition and official certification; the students' privacy is at risk, for the courses and data security solely depend on the centralized online education platform; the students' intellectual property cannot be maintained effectively due to the openness of the Internet and the tamperability of data; there is no mature cross-platform course sharing mechanism to fully share the teaching resources. To make the learning process and results trustable, it is necessary to develop a distributed and trustable data storage method to record the students' learning process, disclose all learning data to the public, and ensure the security and non-tamperability of data.

Blockchain technology is a desirable tool to solve the problems of online education, namely, poor certification, lack of recognition and data insecurity. Currently, this technology is mainly applied in such field as finance, the Internet, and Internet of Things (IoT). Typical financial applications include digital currency, currency transfer, exchange and payment systems. Smart contracts, ranging from securities, stocks to bank loans, can be enforced automatically without human intervention [1]. In terms of the IoT, the blockchain technology enables devices to communicate autonomously and identify errors [2]. This technology has also been applied preliminarily in the field of education. For instance, Mike Sharple proposed that the blockchain can be employed to realize distributed storage of education data, forming the so-called knowledge currency [3]. Some scholars suggested applying blockchain in credit card authentication, secure data encryption and distributed data storage [4]. The MIT Media Lab built a digital learning certificate system using blockchain technology and Mozilla's open badge [5]. In addition, the blockchain technology has been adopted for product design in the industrial field. Sony Global Education [6], a blockchain technology infrastructure platform under Sony Corporation of Japan, can openly and securely share learning courses and record data, without disclosing this information to the education management authority, thus realizing the fairness and digitization of education. University College London uses blockchain technology to help postgraduates of financial risk management verify the authenticity of their academic qualifications [7].

Considering the said problems of online education, this paper explores the nature and features of blockchain technology, and applies this technology in online educa- 
tion, aiming to create a secure, open and trustable online education platform. The research findings pave the way to decentralized development of online education.

\section{Overview of blockchain technology}

The concept of blockchain was invented by a person under the pseudonym Satoshi Nakamoto in his thesis "Bitcoin: A Peer -to -Peer Electronic Cash System" published in 2008 [18]. In this thesis, Nakamoto proposed the Bitcoin system, but did not mention the term blockchain. Instead, the block and chain are described as the data structures to record the history of bitcoin transaction ledger. The "block" refers to distributed data, while the "chain" means the chronological string of blocks arranged by cryptographic methods. Together, the block and the chain constitute a continuous transaction ledger. Broadly speaking, blockchain technology also refers to distributed accounting techniques based on blockchain structure, including distributed consensus, privacy and security protection, peer-to-peer (P2P) communication, network protocols, and smart contracts.

Blockchain technology involves three basic concepts: transaction, block and chain. The transaction is an operation of the ledger like the entry or removal of an item, which always leads to a change in the status of the ledger; the block records the results of all transaction data over a period; the chain is a chronological string of blocks that reflect all the state changes of the ledger.

Blockchain technology is implemented in the following manner. First, there must be a distributed ledger in the network that only allows addition of new data. In other words, no data could be deleted from the ledger, thus ensuring the non-tamperability of the data. As shown in Figure 1, the blocks are linked into a chain in chronological order, with each block retaining the Hash value of the previous block. When a new ledger transaction occurs, the entire system will record the block of the transaction data, and link it to the chain via the elliptic curve digital signature algorithm (ECDSA) [9] in cryptography. In this way, the data cannot be falsified or forged. Meanwhile, the transaction data will be broadcasted across the network, and confirmed by all network nodes, making them undeletable.

Therefore, the blockchain technology has a decentralized, de-trusted, distributed data storage structure. Using cryptographic techniques, this technology guarantees that the data of any transaction cannot be tampered and can be backtracked and verified. The distributed and decentralized nature is protected by the distributed data storage and collective maintenance across the network. Compared with the traditional centralized databases, the blockchain technology eliminates the possibility of the data loss on the entire network caused by the attack on a single node.

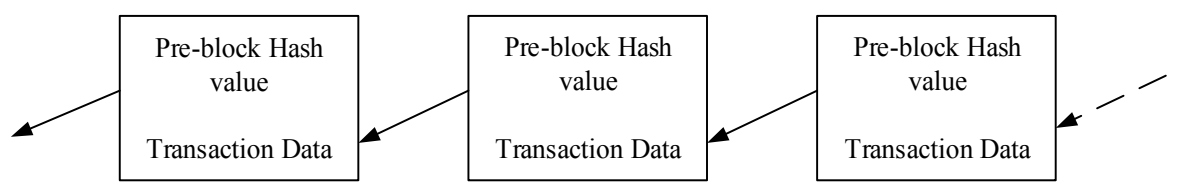

Fig. 1. Blockchain structure 
In general, blockchain technology is decentralized, de-trusted, reliable, collectively maintained, and privacy safe.

1. Decentralized: Relying on decentralized, distributed P2P network [10], the blockchain technology does not need a central node to verify and supervise transaction data. The network nodes can directly exchange data based on the trust system, which improves the efficiency of data exchange. Meanwhile, the damage of a single node will not affect the data of the whole network.

2. De-trusted: The blockchain technology, on the one hand, creates the linked block based on the cryptographic Hash value, and, on the other hand, ensures the transaction safety using the digital signature generated from asymmetric cryptography. Hence, the nodes can make transactions safely without third-party supervision.

3. Reliable: The blockchain database adopts distributed storage, that is, each node can obtain a copy of all transaction data. This storage mode protects the integrity and reliability of the data. Moreover, any transaction data are recorded based on timestamps, and traceable to the very source, indicating that the data are nontamperable.

4. Collectively maintained: The blockchain data are collectively maintained by all nodes in the network. Since no node is excluded from the maintenance, the error of a single node has no impact on the data of the whole network.

5. Privacy safe: Due to the digital signature algorithm, the data are transmitted using the public key and the private key, without disclosing the node identities. The user is completely invisible in the transmission process.

With high credibility and security, the blockchain technology provides an ideal solution to the problems of online education. Specifically, the blockchain can offer complete and non-tamperable learning records for online education, without the need for third-party supervision, and guarantee the fair certification of course credits. Besides, smart contracts can bolster the efficiency of course sharing in online education; cryptographic-based data processing protects the users' privacy. Therefore, it is a promising attempt to apply the blockchain technology in online education.

\section{Application of blockchain technology in online education}

As mentioned above, the technical features of the blockchain can inspire a set of good solutions to the problems of online education. This paper attempts to implement blockchain technology in the following aspects of online education.

\subsection{Full record of learning trajectory}

The blockchain stores data in a distributed database, and records data blocks in chronological order by timestamps. The new data blocks cannot be deleted. The cryptographic algorithm is adopted to prevent the tampering of data, adding to the difficulty in fraud. Currently, most online education platforms are decentralized, offering courses of inconsistent qualities. What is worse, the learning results lack public 
recognition due to the lack of a unified certification system. Unsurprisingly, online education fails to yield fruitful results. The chronological data recording of the blockchain provide a good way to record the learning data of online education.

The students' learning data, including learning time, course files and test results, can be recorded on the blockchain in chronological order, and each data record can be marked with a timestamp. The data accuracy is protected by the cryptographic-based recording method, eliminates the risks like tampering or deletion. Thanks to the decentralization, distributed database and collective maintenance of the blockchain, any education platform or organization will be able to record the learning trajectories of students across regions and time. This will improve platform efficiency and reduce the hardware cost.

Besides fully recording students' learning data, the blockchain-based learning record prevents tampering and deletion, offering a good guarantee to the credibility of students' learning data. At the same time, the learning data, whose reliability is ensured by the encryption technology, can be broadcasted across the network, and easily downloaded by the employer. From the blockchain-based data, the employer can learn more about the learning state of the students and verify their information. Therefore, blockchain technology can effectively avoid paper fraud, fake academic credentials and other misconduct in higher education, and establish a trusted platform for students, teaching platforms and employers.

\subsection{Trusted certification of learning results}

Despite the immense popularity of online education platforms, the students are not enthusiastic after learning a few courses because the learning results are neither publicly recognized nor officially certified. This is attributable to the lag in pushing forward the certification of learning results. At present, the certification for online education is conducted inefficiently by third-party agencies. This mode cannot meet the needs of the boom of online education in the future. When a student is hunting a job, his/her certificates are archived in the education platform or the school, which will be verified by the employer. If he/she loses a certificate, the student has to go through a complicated and inefficient process to obtain another copy of the certificate from the platform or the school. The blockchain technology, however, provides a simple, efficient solution to certification of learning results, especially academic certification. The students' certificates can be verified easily even if they are lost.

The blockchain adopts an asymmetric encryption algorithm in cryptography to ensure the security and credibility of the data. On this basis, it is possible to design a set of learning results certification system. First, the online education platform or the issuing organization records the learning data of students based on the blockchain technology, including the basic information, the course information, the course scores, the date of issuance, etc., and encrypt the data by the private key of the platform or organization. Then, the encrypted digital certificates are issued to the students and other recipients within the network. In this case, the employer can perform Hash verification of the digital certificates using the public key of the platform or organization. 
The blockchain technology can provide a credible learning results certification system, owing to the non-temperable and cryptographic nature of blockchain data. With this system, the students do not have to worry about the loss of the certificate, the platform or organization can streamline the certificate preparation process, and the employer spends less on the verification of the learning results. Overall, the results of online education can be applied to practice in a more efficient manner.

\subsection{Decentralized sharing of education resources}

Currently, there are many online education platforms, offering diverse courses with rich contents. Nevertheless, the courses are not shared across the platforms, due to the constraints like education mode, copyright, etc. For those learning different types of courses, the user experience is rather poor because they have to log into different platforms. Similarly, it is very difficult for students of higher learning to study the knowledge in another school or discipline. Many quality course resources are wasted because of the absence of unified and efficient utilization. With the rise of the sharing economy (e.g. shared bikes), the society is calling for better utilization of resources. In the education field, resource sharing marks the future direction of development. Blockchain technology makes it possible to realize resource sharing in online education.

As a typical application of blockchain technology, smart contract is a program system developed on cryptographic security mechanism. It can complete complex transaction operations without human intervention. The system also supports automatic execution and automatic verification. Smart contract technology can simplify the transaction process, realize smart, automated and decentralized transactions and improve the transaction security [11].

Smart contract preludes the formation of a huge resource sharing platform for online education. Based on smart contract, the online education platform can complete course purchase, settlement and acceptance efficiency and accurately without incurring any labour charges. The distributed storage and collective maintenance of blockchain allows the students to acquire the resources of different platforms by logging into only one node in the blockchain network. Furthermore, the education resource data will not be invalidated when individual nodes are damaged in attacks, which is a strong guarantee of data security. In addition, global knowledge systems like Wikipedia, research institutions, academic journals and other education data can be added to the blockchain network using blockchain technology, creating a global knowledge base. The nodes in any blockchain network can access these knowledge resources. This greatly improves the learning efficiency and enriches the learning methods.

\section{Conclusions}

With the development of Internet technology, online education has become a new way for people to acquire knowledge. However, online education still faces such 
problems as the lack of results certification, poor privacy and the absence of sharing mechanism. As an emerging computer technology, blockchain has been widely adopted in various fields due to the decentralized, de-trusted, reliable features. Therefore, this paper combines the blockchain technology with online education to solve the said problems, forming a smart, decentralized and sharing online education system. The research findings mark the development trend of online education.

\section{$5 \quad$ References}

[1] Kosba, A., Miller, A., Shi, E., Wen, Z., Papamanthou, C. (2016). Hawk: The Blockchain Model of Cryptography and Privacy-Preserving Smart Contracts. Security \& Privacy, 839858.

[2] Emilie, H. (2016). Investigating the potential of blockchains, 12: 11.

[3] Sharples, M., Domingue, J. (2016). The Blockchain and Kudos: A Distributed System for Educational Record. Reputation and Reward. Open Research Online, 40-46.

[4] Devaney L. (2016). 5 things to know about blockchain technology, 12: 11.

[5] Redman J. (2016). MIT Media Lab uses the bitcoin blockchain for digital certificates, 12: 11.

[6] (2016). Sony Global Education Develops Technology Using Blockchain for Open Sharing of Academic Proficiency and Progress Records, 2: 16.

[7] (2018). University College London using Bitcoin Verification to overcome cv fraud, 1: 29, http://blockchain.cs.ucl.ac.uk/press-release/

[8] Satoshi Nakamoto S. (2016). Bitcoin: A peer-to-peer electronic cash system, 11: 29.

[9] Johnson, D., Menezes, A., Vanstone, S. (2001). The Elliptic Curve Digital Signature Algorithm (ECDSA). International Journal of Information Security, 1(1): 36-63. https://doi.org/10.1007/s102070100002

[10] Waterhouse, S., Doolin, D.M., Kan, G., Faybishenko, Y. (2002). Distributed Search in P2P Networks. IEEE Internet Computing, 6(1): 68-72. https://doi.org/10.1109/4236.978371

[11] Morrison, A. (2016). Blockchain and smart contract automation: How smart contracts automate digital business, 1: 7 .

\section{Authors}

Han Sun received the B.S. degree in Computer Science and Technology in 2006 from Nankai University, Tianjin, China. He has been on the faculty of The Army Infantry Academy of PLA, Shijiazhuang 050083, China since 2008. He has been a cisco certified network associate (CCNA) since 2012. His research interests include network technology and educational technology.

Xiaoyue Wang received a master's degree in pedagogy in 2012 from Southwest University, Chongqing, China. She majors in the preschool education, and her research interests include preschool teacher training and early learning. She has been working at Shijiazhuang Preschool Teachers College, Shijiazhuang 50082, China.

Xinge Wang received a master's degree in theoretical physics in 1994 from Hebei Normal University, Shijiazhuang, Hebei, China. He currently teaches at the National 
Defense University of PLA, Beijing 100091, China as a professor and a doctoral tutor. His current research direction is educational technology.

Article submitted 31 July 2018. Resubmitted 02 September 2018. Final acceptance 15 September 2018. Final version published as submitted by the authors. 